\title{
Enhancement of cutting tool surface coating quality using ionized gaseous medium (IGM)
}

\author{
S. O. Yakubu \\ Department of Mechanical Engineering, Nigerian Defence Academy, Kaduna.
}

Accepted 20 December, 2010

\begin{abstract}
The use of ionized gaseous medium (IGM) to prepare a hard alloy material surface by grinding and its effect on the coating quality was investigated. During grinding, IGM was fed to the cutting area by different methods namely: clockwise (longitudinal), anticlockwise (opposed) and transverse (Cross) feeding, respectively. Thereafter, the samples were coated with a titanium nitride on a modern vacuum, ionizing apparatus HHB-6.6-u1 by physical vapour deposition (PVD) method, known as condensation and ionized bombardment (CIB). The analysis of the results and tests carried out revealed that IGM improves the quality of coating, especially when IGM was fed anticlockwise and when the corona discharged current $\left(\tau_{k}\right)$ was equal to $50 \mu \mathrm{A}$. The lowest component forces were also gotten by anticlockwise feeding for example, the component forces $\left(P_{y} P_{z}\right)$ were 2 times and 1.5 times lower compared to longitudinal and cross feeding of IGM, respectively. The micro hardness of inserts ground with IGM was about $10 \%$ higher than those ground with other types of fluids. The micro photograph of inserts structure revealed a distinct and better coated layer for the inserts surfaces prepared prior to coating with IGM. Whereas inserts prepared with compressed air and without cutting fluids showed very blur and indistinct coated layer. It was established that inserts whose surfaces were prepared with IGM and then coated with the titanium nitride (TiN) showed tool-life of about 4 times greater than others.
\end{abstract}

Key words: Ionized gaseous medium, hand alloy tool inserts, coating, condensation and ionized bombardment, titanium nitride.

\section{INTRODUCTION}

The high rate of cutting tools wear during machining (that is, during metal cutting operations, especially during rough machining). It has led to a constant high demand for tools with high wear resistance. This is more pronounced in railway industries (companies) where large quantity of metal layers is removed from the rail wheel-pairs surface everyday. There are many ways of increasing the cutting tool war-resistance (tool life). Some of these ways are the use of cutting fluids, coating the tools surfaces, optimizing the methods of re-sharpening the tool cutting edge(s), etc. However, coating enhances the tool life more than any of the other method (Anikeev et al., 1980).
Irrespective of the type of coating employed, the quality of the coating (that is, adhesiveness of the coating on the surface matrix of the material being coated) depends largely on the quality of the material surface. Therefore, the hard alloy material surface preparation prior to coating deposition is vital.

IGM works on a simple basic principle of drawing atmospheric air through a compressor and the compressed air is passed through a dry electrostatic cutting tool cooling apparatus "Varkash" where it is ionized. The ionized air is then passed to the cutting area (Yakubu and Popov, 2003).

There are two main groups of coating methods namely;

E-mail: ysochetengwu@yahoo.com. Tel: 08028271895. 
the chemical vapour deposition (CVD) and the physical vapour deposition (PVD). There are varieties of CVD methods for example, in Russia, there is the thermogaseous technology (GT). In some countries like Germany, USA, Sweden, Australia, Japan, etc. 60-80\% of their hard alloy tools are coated with CVD method (Samoilov et al., 1988).

Major world producers of had alloy tools like Kennametal-Hertel in Germany, Corboloi (USA), Sandviccoromant in Sweden, Planzee in Australia, Widia (Germany) and Mitsubishi Carbide (Japan), etc. have designed a new technology of coating based on CVD principle. The temperature range for CVD method is 1000-1100 (Lenskaya et al., 1982).

The principle of PVD is based on vapourization of cathode substances in a vacuum of coating deposition apparatus with a simultaneous feeding of reactive gas for example, $\mathrm{N}_{3}, \mathrm{O}_{2}, \mathrm{CH}_{4}$, etc. There are different types of PVD methods:

1. Methods based on condensation of substances from plasma phase in a vacuum with ionized bombardment for example, CIB in Russia and ion bond in USA.

2.Magneto-electronic ionized spurting (MIS) in Switzerland and its Russian variation, Magnetic Reactive Spurting (MARS).

3. lonized cladding in Russia.

The ability to control the temperature of the coating zone in PVD makes it possible to coat not only hard alloy materials, but also high speed steels. PVD is a more common and universal method of coating than CVD especially for single layer, multilayer and composition coatings based on nitrides, carbides, carbonitrides, oxides, boridize of metals in groups IV-VI of the periodic table.

CIB's principle of operation is based on the generation of coating substances with cathode spot (flux) using a highly accurate low voltage vacuum arc discharge exclusively in the cathode material vapour. The feeding of the vacuum camera of the coating apparatus with reactive gases for example, nitrogen, methane, etc. in an ionized bombardment environment, facilitates a smooth plasmachemical reaction which brings about condensation of coating on the cutting surface of a cutting tool (Andrei, 1980). The evaporating plasma-chemical reactions, ionized bombardment and condensation processes take places in the vacuum camera whose metallic body serves as anode.

This research is aimed at preparing a very good surface for a quality coating using unconventional cutting fluids which is ecologically pure that is, human and environmental friendly.

\section{EXPERIMENTAL PROCEDURES}

\section{Hard alloy inserts surface preparation prior to coating}

The preparation of the hard alloy tool inserts surface prior to coating

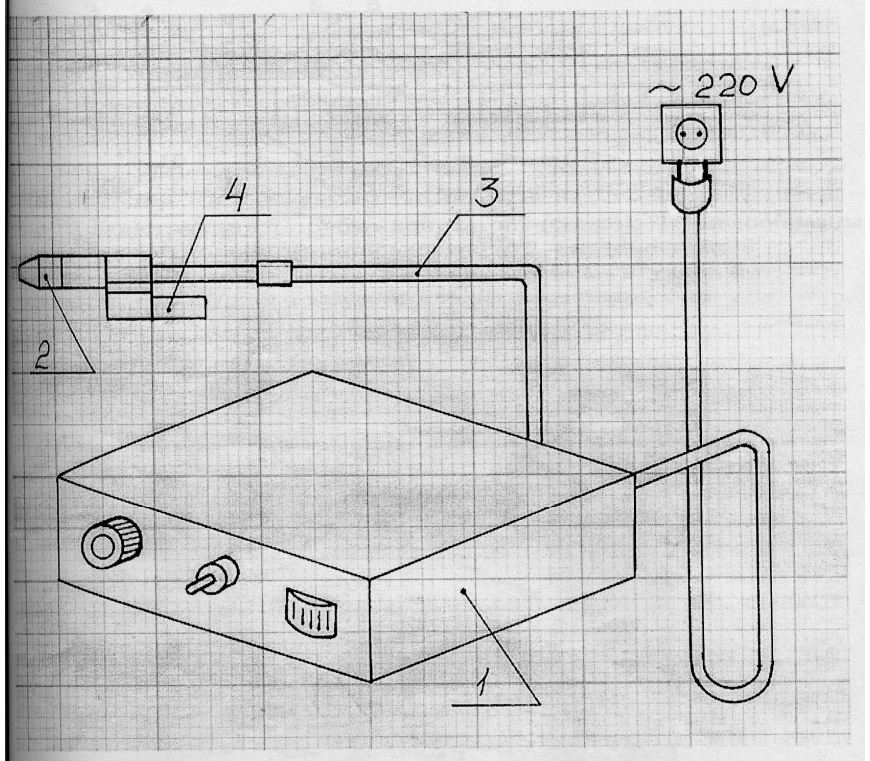

Figure 1. The general view of Varkash. 1- power source, 2nozzle, 3- high voltage connecting cable, 4- "shutser".

was conducted on a flat surface grinding machine using IGM as the cutting fluid. The feeding of IGM to the grinding zone was done with the aid of dry electrostatic cooling apparatus known as "Varkash" (Figure 1). The ionization of the compressed air coming through the compressor was done in the Varkash nozzle with the help of corona discharged current. The hard alloy tool inserts used were from the following grades of hard alloys; T14K8 and T5K10.

In order to improve the efficiency of IGM, it was optimized by feeding it to the grinding area through three different directions namely; longitudinal (clockwise), cross (traverse) and anticlockwise feeding and then, the most effective feeding direction was determined. The values of the corona discharge current were varied from 25 to $100 \mu \mathrm{A}$. The grinding was carried out under the following conditions (Regime): Grind speed $=30 \mathrm{~m} / \mathrm{s}$, work piece speed (that is, cross feeding) $=2.5 \mathrm{~mm} /$ stroke, longitudinal feed $=10 \mathrm{~m} / \mathrm{min}$ and compressed air pressure $=0.3 \mathrm{MPa}$.

\section{Coating deposition process with titanium nitride}

Wear resistance coating was carried out on the surface of hard alloy tool inserts after they were been grounded using IGM. The type of coating deposition employed was PVD using a plasma condensation and ionized bombardment method (CIB). CIB comprises of two sequential processes that is, ionized bombardment and condensation of coating. CIB principle of operation is based on the generation of coating substance with cathode flux in a high accurate low voltage cathode material vapour (5-7).

The titanium Nitride ( $\mathrm{TiN}$ ) coating was conducted on a modern ionized vacuum equipment HHB-6.6-U1 (Figure 2). This apparatus can be used to coat cutting tools surfaces with diameter up to 200 $\mathrm{mm}$ and length of $250 \mathrm{~mm}$ for both hard alloy and HSS materials.

The cathode material used was a titanium evaporator BT1-00GOST19867-74 by the Russian National Standard. Before the titanium nitride coating started, the hard alloy inserts (samples) underwent pneumatic treatment and degreasing (extraction of grease). This operation was done on a vibrating apparatus called "Vibrint", for $2.5 \mathrm{~min}$ with a pressure of $0.1-0.2 \mathrm{MPa}$ until tool nose radius of $0.03-0.04 \mathrm{~mm}$ was achieved, then the samples were put 


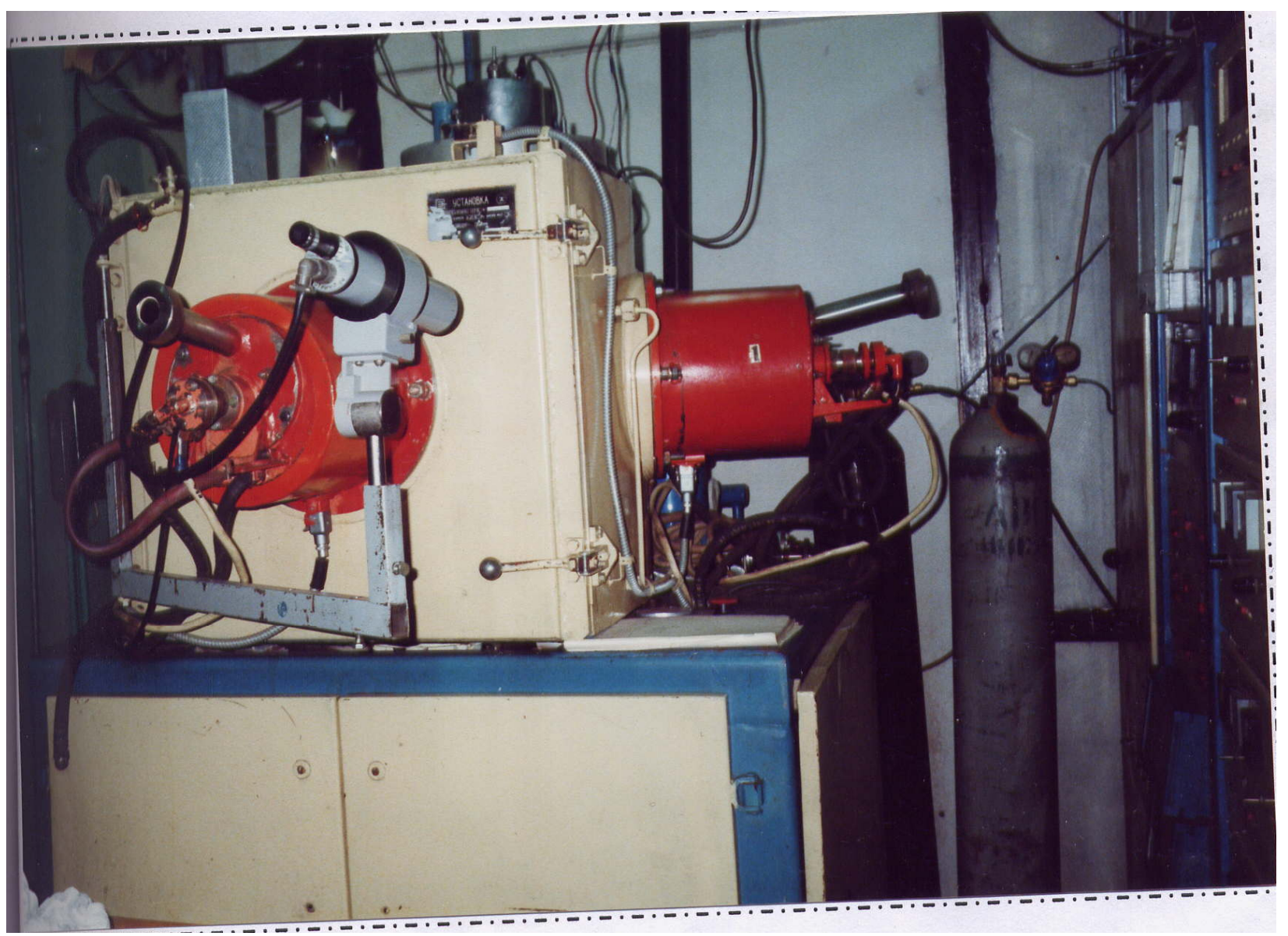

Figure 2. lonized vacuum coating apparatus HBB-6.6-U1.

into the vacuum camera of ionizing apparatus where preliminary ionized cleaning of the samples within temperature of up to $700^{\circ} \mathrm{C}$ was conducted. After the ionized cleaning of the samples, the evaporators were put off and allowed to cool to the desired condensation temperature and then put on again to do coating condensation on the samples surfaces. After the coating condensation process, the evaporators were off again and reactive gas $\left(\mathrm{N}_{2}\right)$ was fed into the vacuum camera with residual pressure of $133 \times 10^{5} \mathrm{~Pa}$.

The residual pressure in the camera was created and controlled from the control board of the coating apparatus. Though the hard alloy tool inserts temperature, the amount of gas fed in and the management of the whole process was done from the control board.The samples surface coating with TiN was executed under the following conditions:

The evaporators' arc current

The pressure of nitrogen

The tool voltage

The hard alloy inserts tension Coating time

The chemical composition of the TiN coating was varied by regulating the arc current of the titanium evaporator, pressure of nitrogen and voltage on the hard alloy tool inserts. The coating quality analysis on the hard alloy tool inserts surface was carried out using an integral evaluation of quality parameters (Vereschaka and Tretyakov, 1986). The use of this method allows in totality to define and evaluate the main parameters of coating such as adhesive strength of the coating to surface matrix, brittle strength of coating, surface roughness, micro hardness and coating thickness. The quality of coating and its adhesiveness to the surface matrix of the hard alloy inserts surface was determined by the formation of "brittle" cracks after the application of a diamond indenter with a force of $600 \mathrm{~N}$ on Rockwell hardness tester TK-2. The test was carried out on three different points on each of the coated insert. The micro hardness of the coated hard alloy inserts were defined using an ultra micro hardness tester "micro Duromat 400" made by Reichert Jung, USA. A diamond indenter with a load of $100 \mathrm{~g}$ was used. The test was carried out on seven different points on the coated hard alloy insert surface, other various tests were carried out on the coated inserts such as, microphotograph of their structures, influence of IGM feeding method on the component forces of grinding, influence of IGM on the wear resistance (that is, tool life) of the hard alloy materials. The last tests was done on a universal lathe machine by turning a low carbon steel where the coated hard alloy inserts served as the cutting tool.

\section{RESULTS AND DISCUSSION}

\section{The effect of IGM on the quality of coating}

The result of the test carried out by the method which 


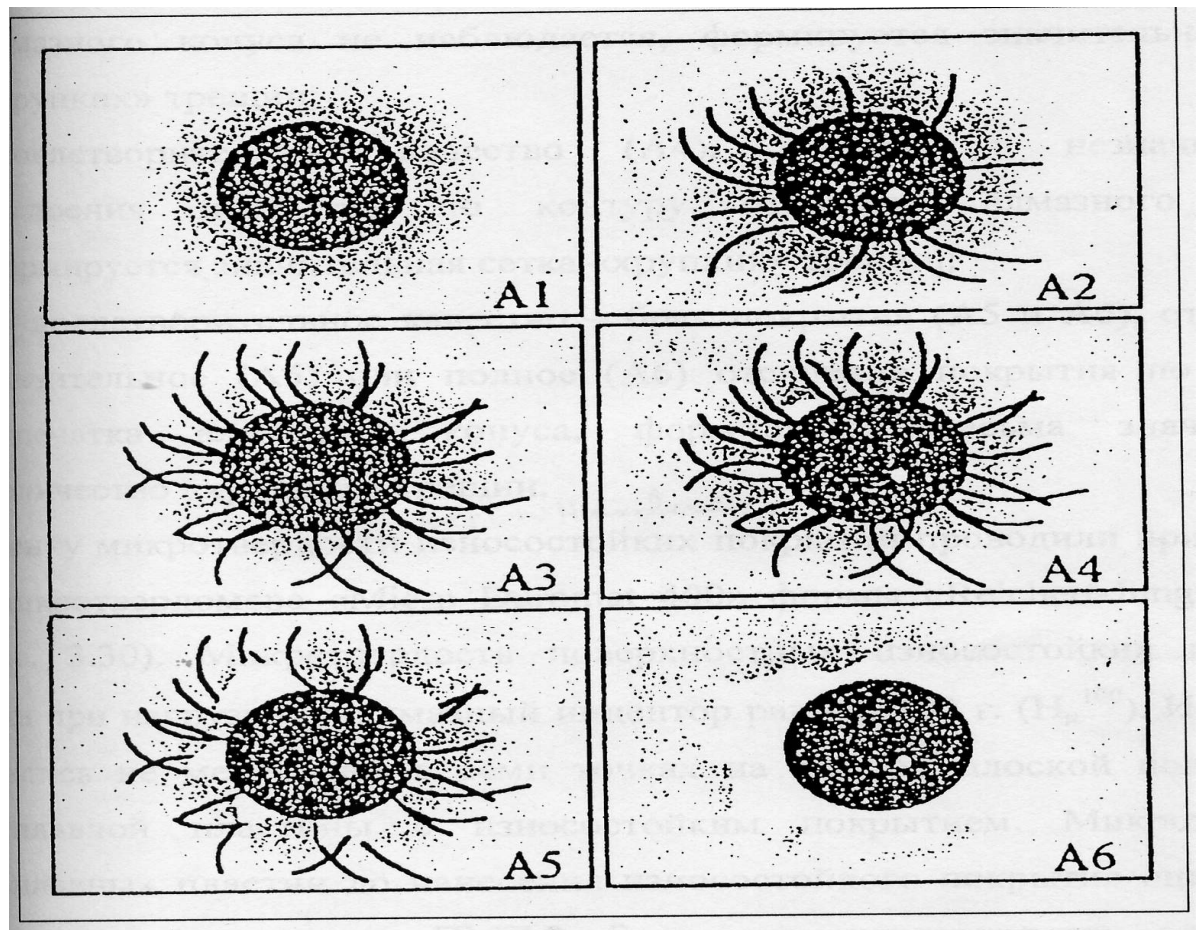

Figure 3. Evaluation scale of surface coating quality of hard alloy tools

was earlier on described in the experimental process is presented in Figure 3 . The black circle is the imprint left by the diamond indenter on the coated hard alloy material surface. The lines are micro-cracks resulting from the impact force of the indenter.

Diagram $A_{1}$ and $A_{2}$ are samples (Figure 3) whose surface were grounded with anticlockwise feeding of IGM. $A_{3}$ and $A_{4}$ are inserts whose surfaces were grounded with longitudinal (clockwise) feeding of IGM and lastly $A_{5}$ and $A_{6}$ are inserts whose surface were grounded with traverse feeding of IGM.

Samples $A_{1}$ showed a very high coating quality and there was no sign of exfoliation or formation of micro cracks around the indenter's imprint. Samples $A_{2}$ have good coating quality because there was no observation of coating exfoliation, but there were very slight traces of micro cracks. In samples $A_{3}$, an increase in micro racks was observed but no coating exfoliation was found in the samples. The quality of coating in samples $A_{3}$ is considered normal. In samples $A_{4}$, there were a lot of micro cracks and a little coating exfoliation. The coating quality of simple $A_{4}$ is considered fairly okay (satisfactory).

However, the samples $A_{5}$ and $A_{6}$ are of poor quality since there was high level of micro cracks and exfoliation of deposited coating. There was a total coating exfoliation in sample $A_{6}$. Judging from these results, one can conclude that the efficiency of IGM in enhancing the coating quality to a large extent depends on the methods of its feeding during grinding prior to coating. In other words, IGM's cooling, lubricating and penetrating properties were more effective during its anticlockwise feeding. Thus, the best quality surface for coating was obtained by anticlockwise feeding of IGM.

\section{Effect of IGM on micro hardness of the coated inserts}

The results of the micro hardness of two different grades of hard alloys T14K8 and T5K10 showed that all the coated hard alloys inserts had higher micro hardness than those which were neither coated nor ground (that is, master samples). It is worthy to note that among the inserts coated, the micro hardness of those whose surfaces were grounded using IGM was greater than those surfaces that were not grounded.

It was also observed that the micro hardness of hard alloys inserts, whose surfaces were grounded using IGM were generally higher than those grounded with other types of cutting fluids (Figure 4). This high increase in micro hardness of inserts ground with IGM may be associated with the formation of new phase. This shows the importance of preparing (grinding) the insert surface before coating (with wear resistance coating).

Furthermore, the micro hardness of the ground surfaces of coated insert T14K8 was $14 \%$ higher than its ungrounded surface. Whereas the micro hardness of the grounded surface of alloy hard insert T5K10 was 10\% higher than its unground surface. It was established that micro hardness of hard alloy tips (inserts) got from hard 


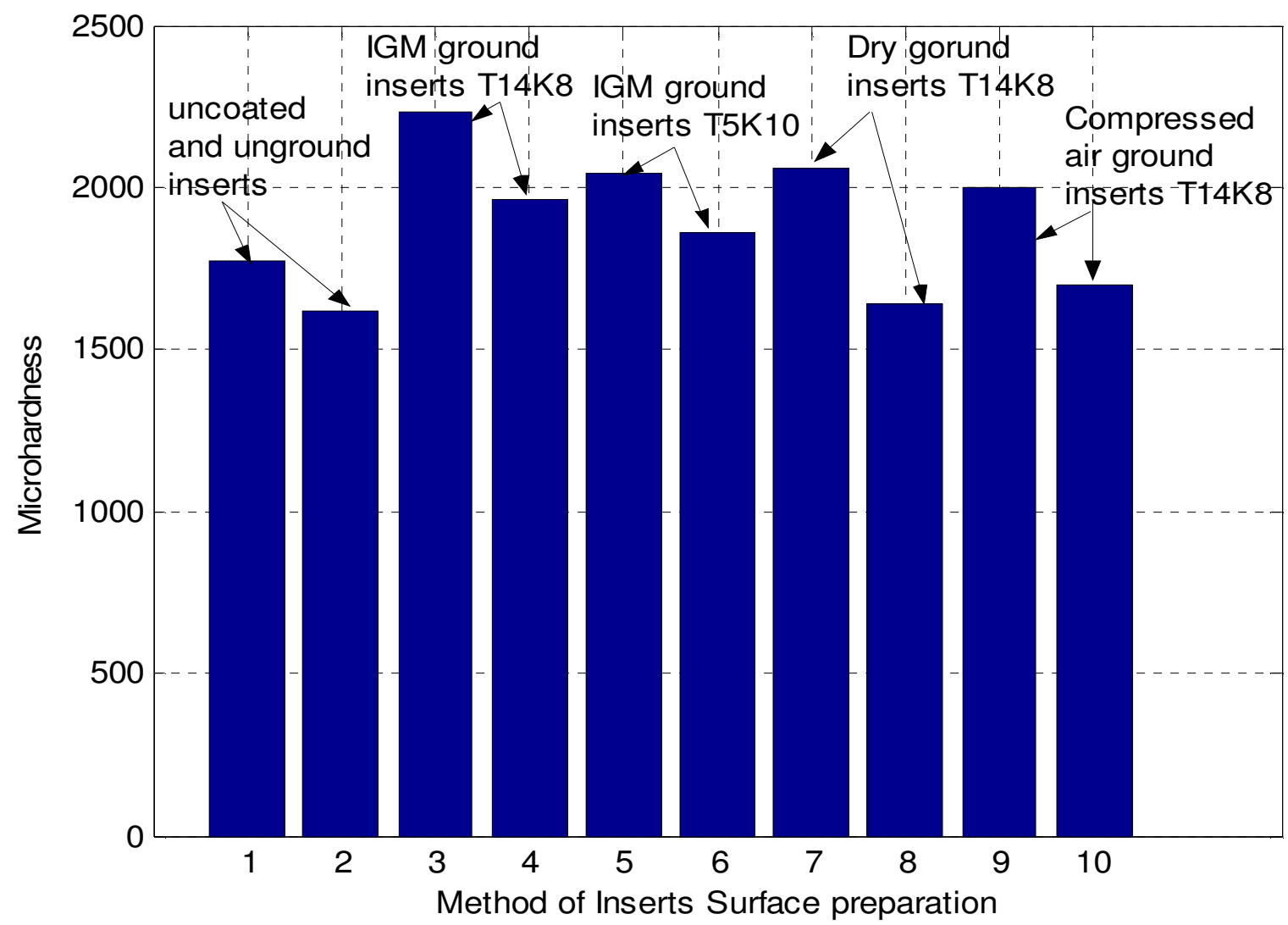

Figure 4. The influence of the surface preparation methods on the coated inserts' micro-hardness $(1,2-$ master samples; 3,4 - ground and unground surfaces T14K8; 5,6 - ground and unground surfaces T5K10; 7,8 - coated and uncoated inserts; 9, 10 - coated and uncoated).

alloy grade $\mathrm{T} 14 \mathrm{~K} 8$ is generally higher than those got from T5K10.

\section{Microstructure of coated insert from hard alloy grade T14K8}

The micro photograph of the structure of the hard alloy insert which is coated with TiN is presented in Figure 5. In the Figure $5 \mathrm{~A}$, is a sample of hard alloy insert ground without the application of cutting fluids; $5 \mathrm{~B}$ is a microphotograph of hard alloy insert ground with IGM, while $5 \mathrm{C}$ is an insert ground with pressurized air. It can be seen from the graph that the three samples have different micro photographic structures. There are three distinct areas in each of the photograph: black, coated layer and base metal. The black part is an empty space, the coated layer indicates the quality of coating and base metal. It was noted that coated layer of the inserts ground with IGM was the most distinct out of the three inserts. This is represented by a very bright white layer between the black part and base metal.

In sample $A$, it is difficult to differentiate between the base metal and the coated layer. In " $\mathrm{C}$ ", coated layer is a bit better, but yet very blur. Thus it is obvious that IGM gives a better coating quality.

\section{Effect of IGM on the hard alloy inserts tool life (wear resistance)}

The efficiency of a cutting tool can be defined through many factors. But the most important is the ability of the cutting tool to resist wearing or failure when it comes in contact with work piece (Yakubu, 2000).

The wearing of a cutting tool is a gradual process subjected to the simultaneous mutual interactions between abrasive, adhesive-fatigue, chemical-oxidizer and diffusion processes (Elgomayel et al., 1979; Gyrevich, 1979). Its effect on tool life and machined surface is negative. Therefore efforts are always made to reduce the tool wearing. The result of the test executed on a TiN coated hard alloy inserts T5K10 was represented graphically in Figure 6, where:

1. Uncoated hard alloy insert from $T 5 K 10$ used to turn low carbon steel with application of cutting fluid (CF).

2. The same uncoated hard alloy inserts but with the 


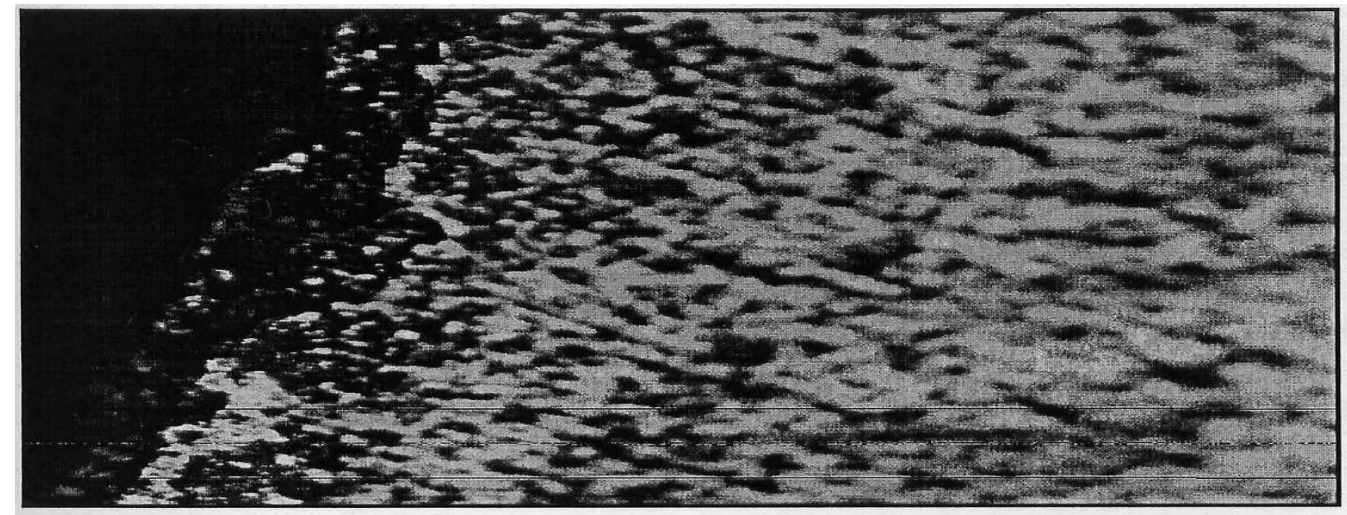

A

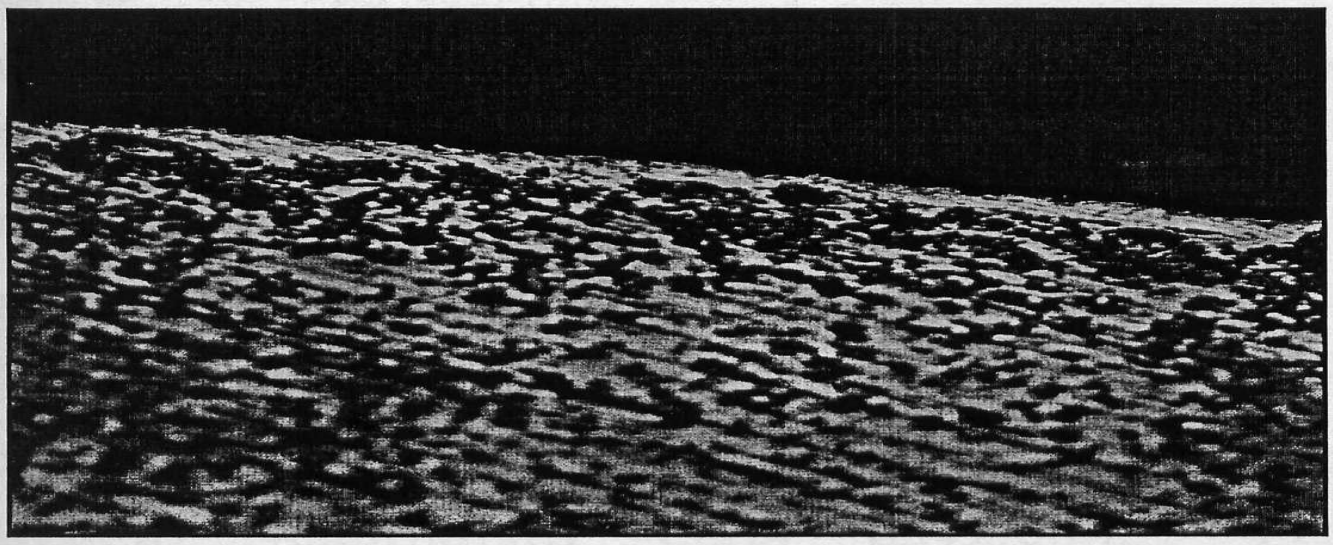

B

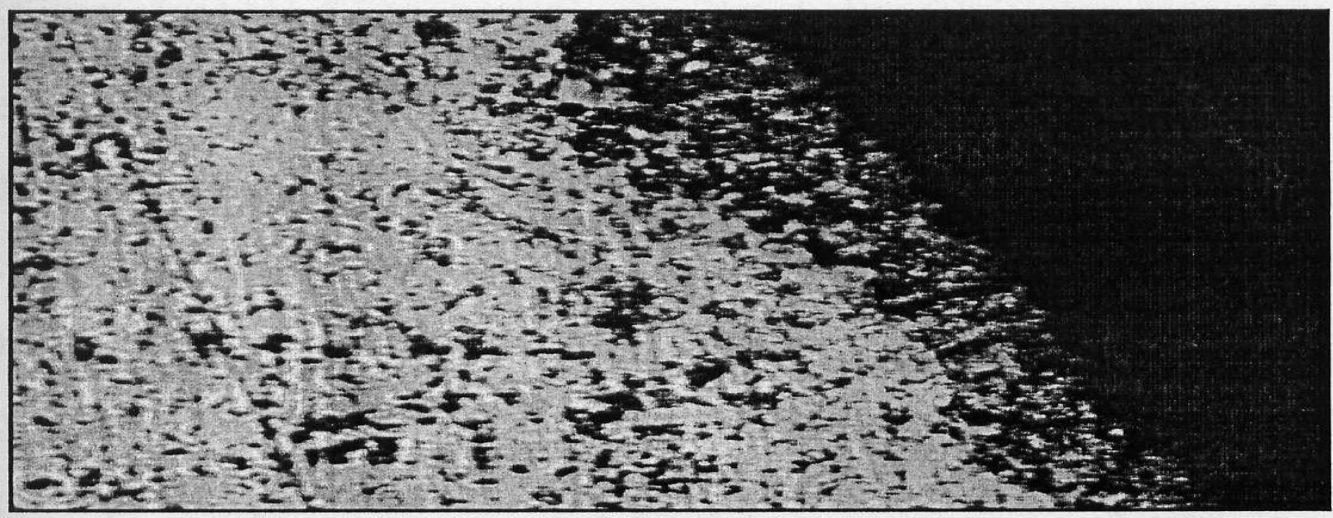

C

Figure 5. The microstructure of hard alloy inserts whose surfaces were grounded using different methods. A , insert 'dry' ground; B, insert ground with IGM; C, insert ground with pressurized air.

application of IGM as CF.

3. A coated inserts under "dry turning".

4. A coated inserts used for turning with the application of IGM.
The result indicates first and foremost, that coating can drastically reduce tool wear (flank wear).Secondly, the use of IGM decreases tool wear even more. The obtained 


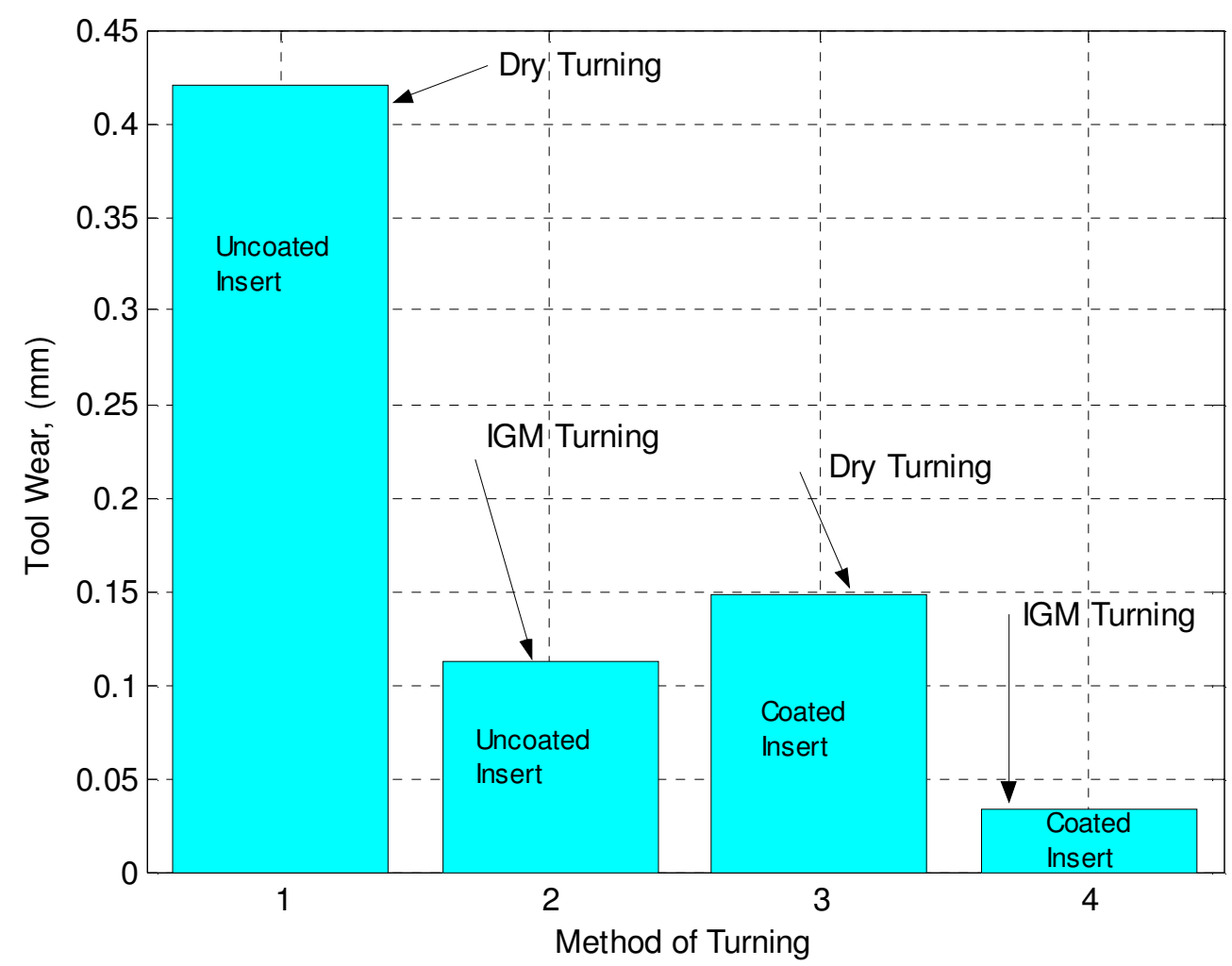

Figure 6. The influence of IGM on the tool surface wears.

Table 1. The influence of IGM on the component forces, during grinding

\begin{tabular}{cccccccc}
\hline & $\begin{array}{c}\text { Corona } \\
\text { S/N }\end{array}$ & \multicolumn{2}{c}{ Anticlockwise feeding of GM } & \multicolumn{2}{c}{ Clockwise feeding of IGM } & \multicolumn{2}{c}{ Cross feeding of IGM } \\
\cline { 3 - 7 } & current $\left(\mathbf{I}_{\mathbf{k}}\right), \boldsymbol{\mu A} \mathbf{A}$ & \multicolumn{2}{c}{ Component forces, $\mathbf{N}$} & \multicolumn{2}{c}{ Component forces, $\mathbf{N}$} & \multicolumn{2}{c}{ Component forces, $\mathbf{N}$} \\
\hline 1 & 25 & $\mathbf{P}_{\mathbf{y}}$ & $\mathbf{P}_{\mathbf{z}}$ & $\mathbf{P}_{\mathbf{y}}$ & $\mathbf{P}_{\mathbf{z}}$ & $\mathbf{P}_{\mathbf{y}}$ & $\mathbf{P}_{\mathbf{z}}$ \\
\hline 2 & 50 & 23.83 & 19.30 & 52.24 & 29.31 & 46.26 & 23.61 \\
3 & 75 & 48.15 & 14.62 & 43.67 & 23.96 & 35.05 & 21.61 \\
4 & 100 & 26.45 & 18.33 & 51.54 & 27.30 & 30.44 & 19.43 \\
\hline
\end{tabular}

result shows that inserts "2" reduction in tool wear is about 4 times compared to insert " 1 " just with application of IGM without coating. With the application of IGM and coating, insert " 4 " indicated a decrease in wear to about 10 times lower than insert " 1 " that is, an increase in tool life of about 10 times greater than inserts "1". Insert "4" has about 4.5 times increase in tool life compared to insert " 3 " and about 4 times compared to insert " 2 ". Thus, the essence of coating and application of IGM cannot be over emphasized.

\section{The effect of IGM on the component forces during grinding}

The effectiveness of IGM does not only depend on the method of its feeding to the cutting zone, but also the corona discharged current. Therefore, IGM efficiency varies according to the value of corona discharged current $\left(\tau_{k}\right)$.

It was noted that IGM was most effective in reducing the component forces (normal and tangential) of grinding when corona discharge current $\left(\tau_{k}\right)$ values were 50 to 75 $\mu \mathrm{A}$ for the three methods of its feeding (Table 1).

However, in anticlockwise feeding, the values of the component forces for all values of $\tau_{k}(25,50,75,100) \mu \mathrm{A}$, are lower than the values obtained from other methods of feeding for example, when the value of $\tau_{\mathrm{k}}=25 \mu \mathrm{A}$, the component forces values for anticlockwise feeding were $P_{y}=39 \mathrm{~N}$ and $P_{z}=19 \mathrm{~N}$. For clockwise feeding, they were 52 and $29 \mathrm{~N}$ and for traverse feeding they were 46 
and $24 \mathrm{~N}$ for normal $\left(P_{y}\right)$ and Tangential $\left(P_{z}\right)$ forces, respectively. When the value of $\tau_{k}=50 \mu \mathrm{A}$ : in the anticlockwise feeding of IGM, $P_{y}=24 N$ and $P_{z}=15 \mathrm{~N}$; in the clockwise feeding, $P_{y}=44 \mathrm{~N}$ and $P_{z}=24 \mathrm{~N}$ and in traverse feeding, $\mathrm{Py}=35 \mathrm{~N}$ and $\mathrm{P}_{\mathrm{z}} 22 \mathrm{~N}$.

The high reduction in the component forces during anticlockwise feeding of IGM was attributed to its high lubricating and cooling performance due to better penetration. As a result, there was drastic reduction in temperature and friction at the contact area.

\section{Conclusion}

Using IGM to grind the hard alloy materials before coating enhances the quality of coating.

The effectiveness of IGM depends both on the method of its feeding and value of corona discharge current. For instance, the best coating quality was obtained under anticlockwise feeding and both the component forces and the tool wear were very small under this feeding method and with the corona discharged current $\left(\mathrm{J}_{\mathrm{k}}\right)=50 \mu \mathrm{A}$.

It was noted that the micro hardness of those hard alloy tools grounded with IGM were higher than those grounded with other methods (compressed air and dry grinding) for example, inserts ground with IGM had micro hardness of 15 and $12 \%$ greater than those grounded with compressed air and dry grinding, respectively.

The microphotograph of the structure of the coated inserts indicated that inserts whose surface were grounded with IGM prior to coating have better quality layer, that is, good clinginess to metal base and distinct coated layer from base metal. It was established that the micro hardness of hard alloy tool from grade T14k8 are generally higher than those from grade T5K10.

\section{RECOMMENDATION}

During grinding, IGM should be fed anticlockwise with corona discharged current $\left(\tau_{k}\right)$ value $=50 \mu \mathrm{A}$ and for the other methods (longitudinal and traverse feedings). $\tau_{k}$ $=100 \mu \mathrm{A}$ for longitudinal and $\tau_{\mathrm{k}}=50-75 \mu \mathrm{A}$ for traverse feedings, respectively. Better quality single layer coating is better done using CIB method on HH6.6U1 apparatus under the following conditions: the evaporators' arc current $=100 \mathrm{~A}$, the pressure of nitrogen $=0.2 \mathrm{~Pa}$, coating time $=20 \mathrm{~min}$

\section{ACKNOWLEDGMENTS}

I say thank you to Engr. C.O. Izelu and Dr. M.Y. Onimisi for taking time to go through this work and making useful suggestions. My profound gratitude goes to the Prof. A.S. Vereschaka for his professional advice and the entire staff of the department of High Effective Technologies, Moscow State University of Technology, STANKIN, Russia for their assistance in conducting the experiments.

\section{ABBREVIATIONS}

IGM, lonized gaseous medium; PVD, physical vapour deposition; TiN, titanium nitride; CIB, condensation and ionized bombardment; CVD, chemical vapour deposition.

\section{REFERENCES}

Andrei AAG IV (1980). The study of some condensers' properties (Ti-N, $\mathrm{Zr}-\mathrm{N})$ obtained through CIB method in a book. Phys. Chem. treatment materials, pp. 64-67.

Anikeev AI, Anikin VN, Toropchenov VS (1980). coating as ways of increasing/improving cutting tools efficiency in a book "Modern hard alloy tool and its rational application" Leningrad, pp. $40-44$.

Elgomayel JI, Radovich JF, Tseung MH (1979). The style of wear mechanism of titanium carbide coated tools. Int. J. machine tools design No. 4:205-219.

Gyrevich DM (1979). Wearing of coated hard alloy tool inserts during turning machine production Bulletin No. 6:45-47.

Lenskaya TG, Toropchenkov VS, Anikeev AS (1982). Tungsten free coated hard alloys in a book "Production and Applications of Hard alloys", Metallurgy Publisher, Moscow, pp. $107-109$.

Samoilov ÉF, Éikhmans VA, Fal K (1988). Hard alloy cutting tool for metal cutting-Reference book, machine production publisher, Moscow. P. 368.

Vereschaka AS, Tretyakov IP (1986). Coated cutting tools. Machine production publisher, Moscow, P.196.

Yakubu SO (2000). Coated hard alloy tools efficiency enhancement by optimizing its surface preparation prior to coating, dissertation thesis, Moscow P. 285.

Yakubu SO, Popov AU (2003). Ecological pure Technology of grinding and Turning machine parts, Science week publisher, Moscow pp. XXII-14. 